\title{
Dynamical evolution of axion condensates under stimulated decays into photons
}

\author{
Pierluca Carenza, ${ }^{1,2, *}$ Alessandro Mirizzi, ${ }^{1,2, \dagger}$ and Günter Sigl ${ }^{3, \$}$ \\ ${ }^{1}$ Dipartimento Interateneo di Fisica "Michelangelo Merlin", Via Amendola 173, 70126 Bari, Italy \\ ${ }^{2}$ Istituto Nazionale di Fisica Nucleare_Sezione di Bari, Via Amendola 173, 70126 Bari, Italy \\ ${ }^{3}$ II. Institute for Theoretical Physics, Hamburg University Luruper Chaussee 149, \\ D-22761 Hamburg, Germany
}

(Received 26 November 2019; accepted 28 April 2020; published 12 May 2020)

\begin{abstract}
Dark matter axion condensates may experience stimulated decays into photon pairs. This effect has been often interpreted as a parametric resonance of photons from the axion-photon coupling, leading to an exponential growth of the photon occupation number in a narrow instability band. Most of the previous literature does not consider the possible evolution of the axion field due to the photon growth. We revisit this effect presenting a mean field solution of the axion-photon kinetic equations, in terms of number of photons and pair correlations. We study the limit of no axion depletion, recovering the known instability. Moreover, we extend the results including a possible depletion of the axion field. In this case we find that the axion condensate exhibits the behavior of an inverted pendulum. We discuss the relevance of these effects for two different cases: a homogeneous axion field at recombination and a localized axion clump and discuss constraints that could result from the induced photon background.
\end{abstract}

DOI: 10.1103/PhysRevD.101.103016

\section{INTRODUCTION}

Axions emerge in relation to the strong $C P$ problem of the strong interactions. Indeed, the most elegant solution to this puzzle is based on the Peccei-Quinn (PQ) mechanism [1-4], in which the Standard Model is enlarged with an additional global $\mathrm{U}(1)_{A}$ symmetry, known as the PQ symmetry. The spontaneous breaking of this symmetry leads as associated Nambu-Goldstone boson, the axion: a low-mass pseudoscalar particle with properties similar to those of neutral pions. The axion mass is given by $[5,6]$

$$
m_{a}=\frac{5.7 \mathrm{eV}}{F_{a} / 10^{6} \mathrm{GeV}}
$$

where $F_{a}$ is the axion decay constant or PQ scale. The axion interactions with photons, electrons, and hadrons are also controlled by the PQ constant and scale as $F_{a}^{-1}$. Notably, the two-photons interaction of axions provides the basis for many possible astrophysical implications and

\footnotetext{
pierluca.carenza@ba.infn.it talessandro.mirizzi@ba.infn.it "guenter.sigl@desy.de
}

Published by the American Physical Society under the terms of the Creative Commons Attribution 4.0 International license. Further distribution of this work must maintain attribution to the author(s) and the published article's title, journal citation, and DOI. Funded by SCOAP ${ }^{3}$. experimental search strategies for these elusive particles. It is described by the Lagrangian [7-9]

$$
\mathcal{L}=-\frac{g_{a \gamma}}{4} \phi \tilde{F}^{\mu \nu} F_{\mu \nu}=g_{a \gamma} \phi \mathbf{E} \cdot \mathbf{B}
$$

where $\tilde{F}^{\mu \nu}=\epsilon^{\mu \nu \rho \sigma} F_{\rho \sigma} / 2$ is the dual of the electromagnetic field $F^{\mu \nu}$ and $\phi$ is the axion field. It would allow for production of axions in stars via Primakoff process [10] and for photon-axion interconversions in magnetic fields that make possible direct detection of axions [11].

Apart from the original theoretical motivation, a renewed interest arose toward axions in the recent years since these still elusive particles may play a crucial role in explaining the dark matter puzzle in the Universe [12]. In particular, axions with masses in the range $10-1500 \mu \mathrm{eV}$ would be the dominant cold dark matter component. In this context their main production mechanism would be the nonthermal realignment, and depending on the cosmological scenario there can be some contribution associated with decays of topological defects, like cosmic strings and domain walls [13]. Generic low-mass axionlike particles (ALPs) exhibiting a two-photon vertex, but which do not satisfy Eq. (1) may also be valid dark matter candidates [14]. Despite their small mass, axions and ALPs can be cold dark matter since being produced nonthermally they are nonrelativistic and behave as a classical field, exhibiting the features of a BoseEinstein condensate [15-17].

The presence of a two photon vertex $a \gamma \gamma$ with effective coupling $g_{a \gamma}$ makes possible the direct detection of dark 
matter axions through haloscope methods. Notably, the ADMX [18] experiment consists of a strong laboratory magnet which would couple the dark matter axion field with the electromagnetic field and create microwave radiation at a detectable level. A new variation of this scheme is the dielectric haloscope approach, where the conversion of dark matter axions to microwave photons is enabled by immersing one or more dielectric layers in a strong laboratory field. This has recently been proposed through the MADMAX [19] project. Another recent proposal is based on tunable cryogenic plasma, which enables resonant conversion by matching the axion mass to a plasma frequency [20].

The two-photon vertex would also lead to a spontaneous axion decay. For the Kim-Shifman-Vainshtein-Zakharov model this rate gives [21-23]

$$
\Gamma_{a \rightarrow \gamma \gamma}=\frac{g_{a \gamma}^{2} m_{a}^{3}}{64 \pi}=1.1 \times 10^{-24} \mathrm{~s}^{-1}\left(\frac{m_{a}}{\mathrm{eV}}\right)^{5},
$$

where the first term at the right-hand side is valid for generic axionlike-particles, while the second uses the relation between $g_{a \gamma}$ and $F_{a}$ for QCD axions. Comparing this decay rate with the lifetime of the Universe $4.3 \times 10^{17} \mathrm{~s}$, one would realize that for typical masses of axion dark matter these particles would be stable on cosmological scales. However, the presence of background photon radiation $f_{\gamma}$ might also lead to stimulated axion decay. In rest frame of the axion condensate, assuming an isotropic photon distribution $f_{\gamma}$ much smaller than the axion one $f_{a}$, it reads [24]

$$
\Gamma_{\text {eff }}=\Gamma_{a \rightarrow \gamma \gamma}\left(1+2 f_{\gamma}\right),
$$

which can increase the rate by many orders of magnitudes. It has been recently pointed out that the photons produced by stimulated axion decays would produce a signal detectable at radio-telescopes [24-28]. In particular, an exponential enhancement of the photon occupation number due to stimulated axion decays has been predicted when photons propagate across the axion condensate with frequency close to the axion mass $m_{a}$. This effect has been interpreted as a parametric resonance of photons, leading to an instability in the axion condensate within a narrow frequency band $\omega \sim$ $m_{a} / 2$ [29-32]. Particularly interesting to study this phenomenon are systems with high axion densities, like axion stars and clumps [30,31,33,34]. An intriguing association between the axion instability and evaporation of axion stars to explain fast radio bursts has also been proposed [35]. Stimulated axion decays in superradiant clouds around primordial black holes have also been recently characterized [36-38]. Most of the studies on the subject have focused on the photon exponential growth under the assumption of no depletion of the axion field. A first attempt to characterise a possible depletion of the axion field has recently been presented in $[39,40]$. We devote our work to revisit the issue of the stimulated decays of axion condensates. Starting from the Heisenberg equations for the relevant operators, we present the kinetic equations for the photon-axion system and study their solution, including the dynamical evolution of the axion field. We also discuss possible applications of the axion-photon instability.

The plan of out work is as follow. In Sec. II we revise the basics of spontaneous and stimulated decay of axions. In Sec. III we study the kinetic equations for the stimulated decays of the axion condensate into photons. At first we characterize the instability of the axion system and then we present the general equations including also the feedback on the axion field. In Sec. IV we present two applications of our finding: the case of a homogeneous axion condensate at the recombination epoch and the case of a localized axion clump. Finally in Sec. V we summarize our results and we conclude. It follows an Appendix in which we discuss the conditions for the axion instability in the expanding Universe.

\section{SPONTANEOUS AND STIMULATED AXION DECAYS}

\section{A. Spontaneous decay rate}

At first we recall the derivation of the axion spontaneous decay rate in vacuum $a \rightarrow \gamma \gamma$. It can be obtained from the usual perturbation theory as

$$
\begin{aligned}
d \Gamma= & \frac{1}{2 m_{a}} \frac{d^{3} k}{(2 \pi)^{3} 2 E_{k}} \\
& \times \frac{d^{3} p}{(2 \pi)^{3} 2 E_{p}}\left|\mathcal{M}_{0}(a \rightarrow \gamma \gamma)\right|^{2}(2 \pi)^{4} \delta^{4}\left(p_{a}-k-p\right),
\end{aligned}
$$

where the amplitude for decay is

$$
i \mathcal{M}_{0}(a \rightarrow \gamma \gamma)=i g_{a \gamma} \epsilon_{\nu}^{*} \epsilon_{\lambda}^{*} \epsilon^{\nu \lambda \alpha \beta} p_{\alpha} k_{\beta},
$$

where $p$ and $q$ are the momenta of the two emitted photons and $\epsilon$ are the polarization vectors. Including a factor $1 / 2$ for the phase space of identical particles, one finds

$$
\begin{aligned}
\Gamma_{a \rightarrow \gamma \gamma} & =\frac{1}{2 m_{a}} \frac{1}{8 \pi} \frac{1}{2} \sum_{\text {pols. }}\left|\mathcal{M}_{0}(a \rightarrow \gamma \gamma)\right|^{2} \\
& =\frac{1}{32 \pi m_{a}} g_{a \gamma}^{2} \times 2(p \cdot k)^{2} \\
& =g_{a \gamma}^{2} \times \frac{m_{a}^{3}}{64 \pi} .
\end{aligned}
$$

\section{B. Stimulated decay rate}

In the presence of a photon background the axion decay rate can be enhanced by a stimulation effect. This can be explicitly seen from the Boltzmann equation for the axion density [24] 


$$
\begin{aligned}
\dot{n}_{a} & =-\int d \Pi_{a} d \Pi_{k} d \Pi_{p}(2 \pi)^{4} \delta^{4}\left(p_{a}-k-p\right)\left|\mathcal{M}_{0}(a \rightarrow \gamma \gamma)\right|^{2}\left[f_{a}\left(f_{\gamma}+1\right)\left(f_{\gamma}+1\right)-\left(f_{a}+1\right) f_{\gamma} f_{\gamma}\right. \\
& =-\int d \Pi_{a} d \Pi_{k} d \Pi_{p}(2 \pi)^{4} \delta^{4}\left(p_{a}-k-p\right)\left|\mathcal{M}_{0}(a \rightarrow \gamma \gamma)\right|^{2}\left[f_{a}\left(1+2 f_{\gamma}\right)-f_{\gamma}^{2}\right] \\
& \simeq-n_{a} \Gamma_{a \rightarrow \gamma \gamma}\left(1+2 f_{\gamma}\right)
\end{aligned}
$$

where $d \Pi$ indicates the phase space Jacobian of the axion and photons. In the last term we used the definition of the axion decay rate $\Gamma_{a}=\int d \Pi_{k} d \Pi_{p}(2 \pi)^{4} \delta^{4}\left(p_{a}-k-\right.$ p) $\left|\mathcal{M}_{0}(a \rightarrow \gamma \gamma)\right|^{2} /\left(2 m_{a}\right)$ in the rest frame of the axion condensate. Moreover, we have neglected the inverse axion decay term, proportional to $f_{\gamma}^{2}$, since for the environment we consider $f_{a} \gg f_{\gamma}$. Under this limit, the stimulation factor only depends on $f_{\gamma}\left(p_{a}-k\right)+f_{\gamma}(k)$. Assuming equal photon distributions we get the term $2 f_{\gamma}$. Therefore, we have an effective rate $[33,34]$

$$
\Gamma_{\text {eff }}=\Gamma_{a \rightarrow \gamma \gamma}\left(1+2 f_{\gamma}\right),
$$

where the last term is the stimulation factor due to the background photon radiation. We recall that $f_{\gamma}$ is the photon occupation number which refers to a density per cell of volume $(2 \pi)^{3}$ (in Planckian units) in phase space, while we will indicate with $n_{\gamma}$ and $n_{a}$, respectively the photon and axion number densities per unit volume in the three-dimensional space. From the previous equation one realizes that the effective axion decay rate into photons $\Gamma_{\text {eff }}$ is equal to the spontaneous decay rate $\Gamma$ given by Eq. (7) only if the photon occupation $f_{\gamma} \ll 1$, and thus in particular if the final state photon modes have not been significantly been populated by previous decays. Otherwise $\Gamma_{\text {eff }}$ can become much larger by stimulated emission.

\section{Exponential photon enhancement in the narrow instability band}

Now we will consider the particular case in which the enhancement factor is produced by photons with occupation numbers $N_{\mathbf{k}}=N_{-\mathbf{k}} \equiv f_{\gamma}$, produced by spontaneous axion decays at rest with momentum $|\mathbf{k}| \simeq m_{a} / 2$. In this section we will follow an heuristic derivation that follows the treatment presented in [41] in the context of inflaton decays in cosmological preheating scenarios.

We start by treating the axion and photon fields as a classical fields. The axion field is real. Moreover, we take the axion field as a nonrelativistic condensate. Therefore, taking that most of the axion dark matter is in the zero momentum mode, it reads $[16,17]$

$$
\phi=\phi_{0} \cos \left(m_{a} t\right) .
$$

However, for further purpose, it is useful to express the real axion field $\phi=\phi^{*}$ as nonrelativistic limit of a complex scalar field $[16,17]$

$$
\phi=\frac{1}{\sqrt{2 m_{a}}} \frac{1}{\sqrt{V}}\left[b_{0} e^{-i m_{a} t}+b_{0}^{*} e^{i m_{a} t} .\right.
$$

For a condensate, $b \simeq \sqrt{N_{a}}$. Therefore, $\phi_{0}=2 \operatorname{R} e\left(b_{0}\right) /$ $\sqrt{2 m_{a} V}=\sqrt{2 n_{a} / m_{a}}$, where $n_{a}$ is the axion number density. From the Lagrangian of Eq. (2) it has been shown that working in the Coulomb gauge, where the electromagnetic potential $A^{\mu}=(\varphi, \mathbf{A})$ satisfies the condition $\nabla \cdot \mathbf{A}=0$, assuming an homogeneous axion field one can obtain the equation of motion for the vector potential (see Refs. [42,43] for the general equations of axion electrodynamics)

$$
\ddot{\mathbf{A}}-\nabla^{2} \mathbf{A}+g_{a \gamma} \dot{\phi} \nabla \times \mathbf{A}=0 .
$$

We will consider the axion field as homogeneous also in the presence of a localized clump, assuming that the spatial field variations $|\nabla \phi| \ll \partial_{t} \phi$ as in [30]. Conversely, in the equations one would find a term proportional to the spatial gradient of $\phi$ and to the scalar potential of the electromagnetic field (see, e.g., [44,45]). We remark that for simplicity we are assuming massless photons, considering values of the axion mass much larger than the plasma frequency. Expanding the vector potential into plane waves

$$
\mathbf{A}(t, \mathbf{x})=\sum_{\lambda} \int \frac{d^{3} \mathbf{k}}{(2 \pi)^{3}} \sqrt{\frac{V}{2 \omega_{k}}} \epsilon_{\lambda}(\mathbf{k}) c_{\mathbf{k}, \lambda} e^{i \mathbf{k} \cdot \mathbf{x}},
$$

one finds the following Mathieu equation $[29,30]$

$$
\ddot{c}_{\mathbf{k}, \pm}+k^{2} c_{\mathbf{k}, \pm} \mp g_{a \gamma} \phi_{0} m_{a} k \sin \left(m_{a} t\right) c_{\mathbf{k}, \pm}=0,
$$

where we worked with circular polarizations $(\lambda= \pm)$ expressed in terms of the polarization vectors

$$
\begin{aligned}
\epsilon_{ \pm}(\mathbf{k}) & =\frac{1}{\sqrt{2}}\left(\epsilon_{1}(\mathbf{k}) \pm i \epsilon_{2}(\mathbf{k})\right), \\
\epsilon_{1}(\mathbf{k}) & =(1,0,0) \\
\epsilon_{2}(\mathbf{k}) & =(0,1,0)
\end{aligned}
$$

It is known that the Mathieu equation has instability bands where the solution shows an instability, i.e., an exponentially growing solution. We will formally discuss the instability properties of the Mathieu equation in the next section. Here we present a heuristic derivation. 
From Eq. (14), since $k \simeq m_{a} / 2$, we can obtain the photon dispersion relation

$$
\begin{aligned}
\omega & =k\left[1 \mp 2 g_{a \gamma} \phi_{0} \sin \left(m_{a} t\right)\right]^{1 / 2} \\
& \simeq \frac{m_{a}}{2}\left[1 \mp g_{a \gamma} \phi_{0} \sin \left(m_{a} t\right)\right] .
\end{aligned}
$$

From this equation one obtains that the photons are created within a thin shell of width

$$
\Delta k \simeq g_{a \gamma} \phi_{0} m_{a} \ll m_{a} .
$$

Then, the number of photons created in the momentum modes around $k=m_{a} / 2$ are given by

$$
\begin{aligned}
f_{\gamma}\left(k=m_{a} / 2\right) & =\frac{n_{\gamma}}{4 \pi k^{2} \Delta k /(2 \pi)^{3}} \\
& =\frac{8 \pi^{2} n_{\gamma}}{m_{a}^{2} g_{a \gamma} \phi_{0} m_{a}}=\frac{4 \pi^{2} \phi_{0}}{m_{a}^{2} g_{a \gamma}} \frac{n_{\gamma}}{n_{a}} .
\end{aligned}
$$

The number density $n_{\gamma}$ exceeds unity and so stimulated emission is essential only if

$$
n_{\gamma}>\frac{g_{a \gamma} m_{a}^{2}}{4 \pi^{2} \phi_{0}} n_{a} .
$$

Therefore, from Eq. (9) the effective decay rate reads

$$
\Gamma_{\text {eff }}=\frac{g_{a \gamma}^{2} m_{a}^{3}}{64 \pi}\left(1+\frac{8 \pi^{2} \phi_{0}}{m_{a}^{2} g_{a \gamma}} \frac{n_{\gamma}}{n_{a}}\right) .
$$

Then, the Boltzmann equation for the photons is given by

$$
\begin{aligned}
\dot{n}_{\gamma} & =2 \Gamma_{\mathrm{eff}} n_{a} \\
& =\frac{g_{a \gamma}^{2} m_{a}^{3}}{32 \pi}\left(1+\frac{8 \pi^{2} \phi_{0}}{m_{a}^{2} g_{a \gamma}} \frac{n_{\gamma}}{n_{a}}\right) n_{a} .
\end{aligned}
$$

In the case Eq. (19) is satisfied, the previous equation can be easily integrated, leading to an exponentially growing solution

$$
n_{\gamma}=\exp [\tilde{\mu} t] n_{\gamma}(0)
$$

with growth rate

$$
\tilde{\mu}=\pi \frac{g_{a \gamma} m_{a} \phi}{4} .
$$

Note that in this case of exponential speed-up, the growth rate is proportional to $g_{a \gamma}$ and not to $g_{a \gamma}^{2}$ as in the spontaneous decay.

\section{KINETIC EQUATIONS FOR DECAYING AXION CONDENSATES}

\section{A. Axion-photon Hamiltonian}

In this section we will present the kinetic equations for the decaying axion condensate. Most of the previous works treat the photon field as a classical one, and based their solution on the Lagrangian in Eq. (2). Since we will work out the quantum Heisenberg equations for the ensemble, we will work in an Hamiltonian formalism, inspired by the approach of $[39,40]$. In particular, from the Lagrangian in Eq. (2) the interaction Hamiltonian is

$H_{\mathrm{int}}=\frac{g_{a \gamma}}{4} \int d^{3} \mathbf{x} \phi \tilde{F}^{\mu \nu} F_{\mu \nu}=-g_{a \gamma} \int d^{3} \mathbf{x} \phi \mathbf{E} \cdot \mathbf{B}$.

We will work in the radiation gauge, where the Hamiltonian assumes the form

$$
H_{\mathrm{int}}=g_{a \gamma} \int d^{3} \mathbf{x} \phi \dot{\mathbf{A}} \cdot \nabla \times \mathbf{A}
$$

In order to quantize the Hamiltonian we expand the vector potential in terms of free fields, assuming the interaction is a small perturbation

$$
\begin{aligned}
\mathbf{A}(t, x)= & \frac{1}{(2 \pi)^{3}} \sum_{\lambda} \int d^{3} \mathbf{k} \sqrt{\frac{V}{2 \omega_{k}}}\left(\epsilon_{\lambda}(\mathbf{k}) c_{\mathbf{k}, \lambda} e^{-i k x}\right. \\
& \left.+\epsilon_{\lambda}^{*}(\mathbf{k}) c_{\mathbf{k}, \lambda}^{\dagger} e^{i k x}\right),
\end{aligned}
$$

where now $c_{\mathbf{k}, \lambda}$ is an operator. Note that $V d^{3} \mathbf{k} /(2 \pi)^{3}$ is the dimensionless phase space element that becomes a sum over discrete modes in a finite volume and the operators $c_{\mathbf{k}, \lambda}$ and $c_{\mathbf{k}, \lambda}^{\dagger}$ are dimensionless annihilation and creation operators, respectively, satisfying canonical commutation relations $\left[c_{\mathbf{k}, \lambda}, c_{\mathbf{k}^{\prime}, \lambda^{\prime}}^{\dagger}\right]=(2 \pi)^{3} \delta^{3}\left(\mathbf{k}-\mathbf{k}^{\prime}\right) \delta_{\lambda, \lambda^{\prime}} / V$ which reduce to $\delta_{\mathbf{k}, \mathbf{k}^{\prime}} \delta_{\lambda, \lambda^{\prime}}$ in the discrete case. Treating the axion field as a classical field for now, the quantized interaction Hamiltonian in terms of circular polarizations is then

$$
\begin{aligned}
H_{\mathrm{int}}= & -i g_{a \gamma} \phi_{0} \cos \left(m_{a} t\right) \int_{\Omega} \frac{V d^{3} \mathbf{k}}{(2 \pi)^{3}} \omega_{k}\left[\left(c_{\mathbf{k}, \omega,+} c_{-\mathbf{k}, \omega,+}\right.\right. \\
& \left.\left.-c_{\mathbf{k}, \omega,-,} c_{-\mathbf{k}, \omega,-}\right)-\left(c_{\mathbf{k}, \omega,+}^{\dagger} c_{-\mathbf{k}, \omega,+}^{\dagger}-c_{\mathbf{k}, \omega,-}^{\dagger} c_{-\mathbf{k}, \omega,-}^{\dagger}\right)\right],
\end{aligned}
$$

where $c_{\mathbf{k}, \omega}=c_{\mathbf{k}} e^{-i \omega_{k} t}$ with $\omega_{k}=|k|$. Here the integral over the phase-space only runs over half of the directions. Finally, the total Hamiltonian can be written as 


$$
\begin{aligned}
H= & H_{0}+H_{\mathrm{int}} \\
= & \sum_{\lambda= \pm} \int_{\Omega} \frac{V d^{3} \mathbf{k}}{(2 \pi)^{3}} \omega_{k}\left(N_{\mathbf{k}, \lambda}+N_{-\mathbf{k}, \lambda}\right)-i g_{a \gamma} \phi_{0} \cos \left(m_{a} t\right) \\
& \times \int_{\Omega} \frac{V d^{3} \mathbf{k}}{(2 \pi)^{3}} \omega_{k}\left[\left(C_{\mathbf{k}, \omega,+}-C_{\mathbf{k}, \omega,-}\right)-\left(C_{\mathbf{k}, \omega,+}^{\dagger}-C_{\mathbf{k}, \omega,-}^{\dagger}\right)\right] \\
= & \int_{\Omega} \frac{V d^{3} \mathbf{k}}{(2 \pi)^{3}} \omega_{k} N_{\mathbf{k}}-i g_{a \gamma} \phi_{0} \cos \left(m_{a} t\right) \\
& \times \int_{\Omega} \frac{V d^{3} \mathbf{k}}{(2 \pi)^{3}} \omega_{k}\left[C_{\mathbf{k}, \omega}-C_{\mathbf{k}, \omega}^{\dagger}\right]
\end{aligned}
$$

where we introduced the number

$$
N_{ \pm \mathbf{k}, \lambda}=c_{ \pm \mathbf{k}, \lambda}^{\dagger} c_{ \pm \mathbf{k}, \lambda},
$$

and pair correlation operators

$$
C_{\mathbf{k}, \omega, \lambda}=c_{\mathbf{k}, \omega, \lambda} c_{-\mathbf{k}, \omega, \lambda},
$$

for a given polarization state, as well as the total number operator summed over polarization state

$$
N_{\mathbf{k}}=N_{\mathbf{k},+}+N_{-\mathbf{k},+}+N_{\mathbf{k},-}+N_{-\mathbf{k},-},
$$

and the difference of the pair correlation operators for the two polarization states

$$
C_{\mathbf{k}, \omega}=C_{\mathbf{k}, \omega,+}-C_{\mathbf{k}, \omega,-} .
$$

Note that the number operators $N_{ \pm \mathbf{k}, \lambda}$ do not depend on the photon frequency. The previous operators satisfy the following commutation relations

$$
\begin{aligned}
{\left[C_{\mathbf{k}, \omega}, C_{\mathbf{k}^{\prime}, \omega^{\prime}}^{\dagger}\right.} & =\left(2+N_{\mathbf{k}}\right)(2 \pi)^{3} \delta^{3}\left(\mathbf{k}-\mathbf{k}^{\prime}\right) / V, \\
{\left[N_{\mathbf{k}}, C_{\mathbf{k}^{\prime}, \omega^{\prime}}\right] } & =-2 C_{\mathbf{k}, \omega}(2 \pi)^{3} \delta^{3}\left(\mathbf{k}-\mathbf{k}^{\prime}\right) / V .
\end{aligned}
$$

\section{B. Heisenberg equations}

From the Hamiltonian in Eq. (28) one obtains the following Heisenberg equations

$$
i \dot{c}_{\mathbf{k}, \omega, \lambda}=\left[c_{\mathbf{k}, \omega, \lambda}, H_{0}+H_{\text {int }}\right],
$$

which reads

$\dot{c}_{\mathbf{k}, \omega,-}=-i \omega_{k} c_{\mathbf{k}, \omega,-}-g_{a \gamma} \omega_{k} \phi_{0} \cos \left(m_{a} t\right) c_{-\mathbf{k}, \omega,-}^{\dagger}$,

$\dot{c}_{\mathbf{k}, \omega,+}=-i \omega_{k} c_{\mathbf{k}, \omega,+}+g_{a \gamma} \omega_{k} \phi_{0} \cos \left(m_{a} t\right) c_{-\mathbf{k}, \omega,+}^{\dagger}$.

These equations can be cast under the form of a second order differential equation

$\ddot{c}_{\mathbf{k}, \omega, \pm}+\omega_{k}^{2} c_{\mathbf{k}, \omega, \pm} \pm g_{a \gamma} \omega_{k} \phi_{0} m_{a} \sin \left(m_{a} t\right) c_{-\mathbf{k}, \omega, \pm}^{\dagger}=0$.
Using $c_{-\mathbf{k}, \omega, \pm}^{\dagger}=-c_{\mathbf{k}, \omega, \pm}$ one can rewrite the previous equations as

$\ddot{c}_{\mathbf{k}, \omega, \pm}+\omega_{k}^{2} c_{\mathbf{k}, \omega, \pm} \mp g_{a \gamma} \omega_{k} \phi_{0} m_{a} \sin \left(m_{a} t\right) c_{\mathbf{k}, \omega, \pm}=0$,

which corresponds to the Mathieu equation found before [Eq. (14)]. Here we notice that among the different applications, the Mathieu equations describes an inverted pendulum, driven by an external vertical pivot, oscillating at high frequency (the so called Kapitza's pendulum) [46]. The linearized equation of motion for the angular displacement $\varphi$ is given by

$$
\ddot{\varphi}+f(1-\mu \cos t) \varphi=0
$$

where $\mu$ is the driving amplitude, and $f$ is the driving frequency squared. For such a system, a parametric resonance can cause an instability of the pendulum, when its frequency is a multiple of the driving frequency of the pivot. In our system, the oscillating axion condensate acts as the external pivot driving the instability.

We will find here the exponentially growing solution of the Mathieu equation in the first instability band. In the limit of small amplitude of the photon field, the solution of Eq. (37) can be found analytically through a linearized stability analysis. We closely follow the discussion presented in [30]. The operator $c_{\mathbf{k}, \pm}$ can be expanded as

$$
c_{\mathbf{k}, \omega, \pm}=\sum_{\omega} e^{-i \omega t} f_{\omega, \pm}(t)
$$

where $f_{\omega, \pm}(t)$ is a slowly varying function of $t$ and $\omega$ runs over integer multiples of $m_{a} / 2$. Inserting this expansion into the Mathieu equation (37) we obtain

$$
\begin{aligned}
& 2 i \omega \dot{f}_{\omega, \pm}+\left(\omega^{2}-k^{2}\right) f_{\omega, \pm} \\
& \quad \pm \frac{g_{a \gamma} \phi_{0} m_{a} k}{2 i}\left(f_{\omega-m_{a}, \pm}-f_{\omega+m_{a}, \pm}\right)=0 .
\end{aligned}
$$

Neglecting $\ddot{f}_{\omega}$, the lowest frequency modes corresponds to $\omega= \pm m_{a} / 2$ and give

$$
\begin{aligned}
& \dot{f}_{m_{a} / 2, \pm}+\frac{1}{i m_{a}}\left(\frac{m_{a}^{2}}{4}-k^{2}\right) f_{m_{a} / 2, \pm} \mp \frac{g_{a \gamma} \phi_{0} k}{2} f_{-m_{a} / 2, \pm}=0 \\
& \dot{f}_{-m_{a} / 2, \pm}-\frac{1}{i m_{a}}\left(\frac{m_{a}^{2}}{4}-k^{2}\right) f_{-m_{a} / 2, \pm} \mp \frac{g_{a \gamma} \phi_{0} k}{2} f_{m_{a} / 2, \pm}=0 .
\end{aligned}
$$

An instability is associated with an exponentially growing solution $f \sim \exp [\mu t]$. The parameter $\mu$ is called Floquet exponent. One explicitly finds 


$$
\mu=\sqrt{\frac{g_{a \gamma}^{2} \phi_{0}^{2} k^{2}}{4}-\frac{1}{m_{a}^{2}}\left(k^{2}-\frac{m_{a}^{2}}{4}\right)^{2}},
$$

where the maximum growth obtained for $k=m_{a} / 2$ is

$$
\mu=g_{a \gamma} m_{a} \phi_{0} / 4
$$

The edges of the instability band are given when $\mu=0$ :

$$
k_{L / R}=\sqrt{\frac{g_{a \gamma}^{2} \phi_{0}^{2} m_{a}^{2}}{16}+\frac{m_{a}^{2}}{4}} \pm \frac{g_{a \gamma} \phi_{0} m_{a}}{4},
$$

and the bandwidth is

$$
\Delta k=g_{a \gamma} m_{a} \phi_{0} / 2 .
$$

In Fig. 1 we show the instability band of the Mathieu equation [Eq. (37)] obtaining numerically solving this equation and plotting at late time the behavior of $\left|c_{\mathbf{k}}\right|^{2}$ in function of $k$.

This solution of the Mathieu equation can be interpreted as a parametric resonance, occurring when $\omega=m_{a} / 2$. This growth rate can be compared with the one found in Eq. (23), where we recall that $n_{k} \sim\left|c_{k}\right|^{2}$. One realizes that the two rates agree apart from a numerical factor. This discrepancy is due to the fact that in Eq. (17) the approximation for the instability width overestimated it, producing a larger photon growth rate. From this comparison it results that the stimulated decay discussed in the previous section can be interpreted as narrow parametric resonance of the Mathieu equation, and vice versa.

Finally, we mention that the treatment of the stimulated axion decays in photon, based on the presence of an instability of the Mathieu equation in a narrow band, has been widely discussed in cosmology in the context of reheating after the inflation, associated with the decay of inflatons $\phi$ into two bosons $\chi$, i.e., $\phi \rightarrow \chi \chi$. Indeed, most of the results presented

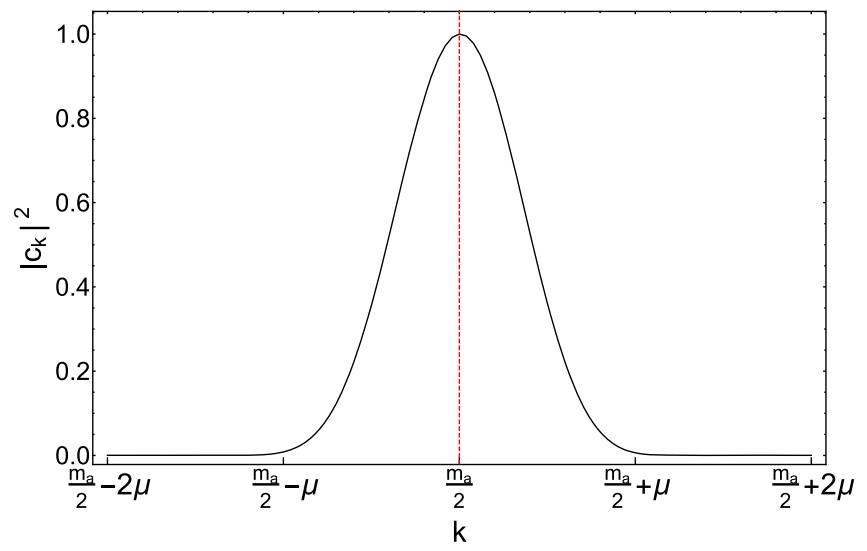

FIG. 1. Instability band of the Mathieu equation [Eq. (37)]. See the text for details. in classical papers on the subjects (see, e.g., [47-51]), can be directly translated to the axion case.

\section{Equations for photon numbers and correlations}

The Mathieu equation allows one to determine the presence of an instability band. However, it does not tell how the instability is triggered. Indeed, one needs to assume a nonzero initial value of the $c_{\mathbf{k}}$ operator in order to have an exponential growth. In the literature, often one introduces the concept of vacuum fluctuations to give an initial seed to $c_{\mathbf{k}}$. However, as pointed out in $[39,40]$, this is not completely satisfactory, especially in a cosmological context where one should invoke a fluctuation coherent on a cosmological scale. Moreover, the operators $c_{\mathbf{k}}$ are not observable. Therefore, it would be more convenient to express the equations of motion in terms of the photon number $N_{\mathbf{k}}$ and correlation operators $C_{\mathbf{k}}$. In order to obtain these sets of equations, we reinsert the definition of the axion field $\phi$ in terms of creation and annihilation operators $b^{\dagger}$ and $b$ [Eq. (11)] in the interaction Hamiltonian $H_{\text {int }}$ of Eq. (27). Moreover, since we will consider only modes in the instability band with $\omega_{k} \approx m_{a} / 2$, removing the rapidly oscillating terms in the Hamiltonian, we get

$$
H_{\mathrm{int}}=-i \frac{\mu}{\sqrt{N_{a}}} \sum_{|\mathbf{k}|=\frac{m_{a}}{2}}\left[b^{\dagger} C_{\mathbf{k}}-b C_{\mathbf{k}}^{\dagger}\right]
$$

where the sum is extended on modes with $|\mathbf{k}|=m_{a} / 2$. From the previous Hamiltonian, working in a corotating reference system with frequency $m_{a} / 2$ we find the following Heisenberg equations

$$
\begin{aligned}
\dot{N}_{\mathbf{k}} & =2 \kappa\left[b^{\dagger} C_{\mathbf{k}}+b C_{\mathbf{k}}^{\dagger}\right], \\
\dot{C}_{\mathbf{k}} & =\kappa\left(2+N_{\mathbf{k}}\right) b, \\
\dot{b} & =-\kappa \sum_{|\mathbf{k}|=\frac{m_{a}}{2}} C_{\mathbf{k}},
\end{aligned}
$$

which agree with the kinetic equation recently presented in $[39,40]$ and where the combination $\kappa \equiv \mu / \sqrt{N_{a}}$ is manifestly independent of $N_{a}$, as it should be for an operator equation involving $b$.

In the following we will consider the solution of the previous system in the mean field approximation, assuming that the previous operators are $c$-numbers. A discussion about effects beyond mean field has been recently given in $[39,40]$.

Note that the Hamiltonian of Eq. (47) has a nice analogy with the trilinear Hamiltonian used in quantum optics to explain spontaneous parametric down-conversion [52-54]. Indeed if we limit ourselves to the case of a single mode with $\omega_{k}=m_{a} / 2$ we get

$$
H_{\mathrm{int}}=\kappa\left[b^{\dagger} c_{\mathbf{k}} c_{-\mathbf{k}}+b c_{\mathbf{k}}^{\dagger} c_{-\mathbf{k}}^{\dagger}\right]
$$


where $c_{\mathbf{k},+} c_{-\mathbf{k},+}-c_{\mathbf{k},-} c_{-\mathbf{k},-} \equiv c_{\mathbf{k}} c_{-\mathbf{k}}$. In the language of quantum optics this Hamiltonian describes the interaction of the pump $b$ with two photons: the signal $c_{\mathbf{k}}$ and the idler $c_{-\mathbf{k}}$.

\section{Short-time evolution}

At first, we consider the short time solution, assuming that the axion field has not been depleted by the decays into photons. In this limit, assuming a constant axion field with $b \approx b^{\dagger} \approx \sqrt{N_{a}}$ the Heisenberg equations for the previous photon number operator and the correlations read

$$
\begin{aligned}
\dot{N}_{\mathbf{k}} & =2 \mu\left[C_{\mathbf{k}}+C_{\mathbf{k}}^{\dagger}\right], \\
\dot{C}_{\mathbf{k}} & =\mu\left(2+N_{\mathbf{k}}\right),
\end{aligned}
$$

where $\mu=g_{a \gamma} m_{a} \phi_{0} / 4$ is the growth rate of Eq. (43), found from the Mathieu equation. It is interesting that in these quantum equations, even we assume that initially there is no radiation photon $N_{\mathbf{k}}=0$, one can still have a nontrivial evolution. Indeed, $\dot{C}_{\mathbf{k}} \neq 0$, since in the last term in the right-hand side of the second equation there is still an initial photon component $\left(2+N_{\mathbf{k}}\right) \simeq 2$ due to the spontaneous axion decay. Even without preexisting photon background, the spontaneous axion decay provides an unavoidable photon seed.

One finds

$$
\ddot{N}_{\mathbf{k}}=4 \mu^{2}\left(2+N_{\mathbf{k}}\right),
$$

whose solution is

$$
N_{\mathbf{k}}(t)=N_{\mathbf{k}}(0) e^{2 \mu t}+2(\cosh (2 \mu t)-1) .
$$

In Fig. 2 we show the behavior of the previous equation as a function of $s=\mu t$, assuming $N_{\mathbf{k}}(0)=0$. It is interesting to notice that for $t \ll \mu^{-1}$ the previous equation has as solution $N_{\mathbf{k}} \sim 4 s^{2}$. This corresponds to the initial phase where the

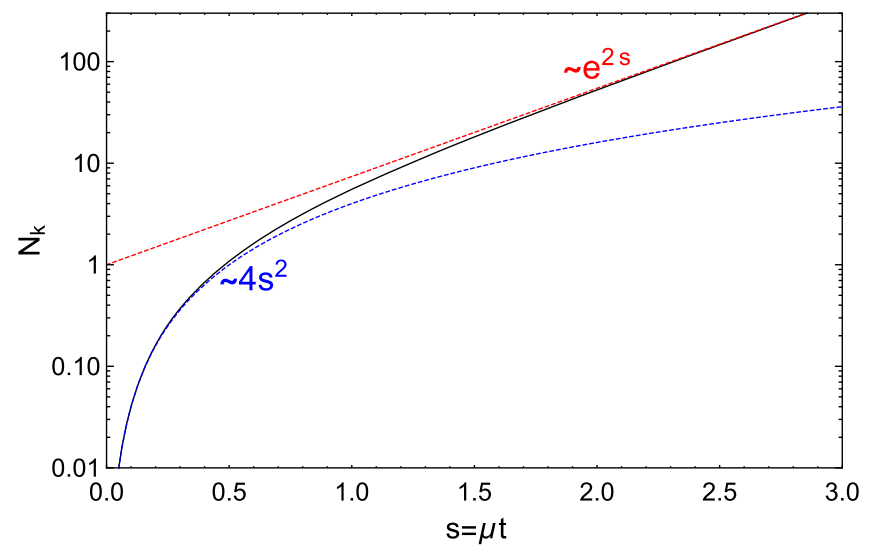

FIG. 2. Short-time solution of axion-photon conversions of Eq. (51) for $N_{\mathbf{k}}(0)=0$. spontaneous decay scaling as $g_{a \gamma}^{2}$ populates the photon field. Instead the long-time solution for $t \gg \mu^{-1}$ scales as $N_{\mathbf{k}} \sim \exp [2 s]$, in agreement with the exponential solution found from the Mathieu equation. In the language of quantum optics this limit corresponds to a parametric amplifier in the limit of a constant pump. In the cosmological environment, which is the natural case for the effect we are studying, there are many sources of photons with energy $\omega=m_{a} / 2$ which can trigger the stimulated axion decays, e.g., a fraction of the photons of the cosmic microwave background or a fraction of the photons in normal matter at some temperature will have some photons of energy $\omega=m_{a} / 2$. Therefore, the presence of an initial photon seed $N_{\mathbf{k}} \neq 0$ is guaranteed. Nevertheless, it is intriguing to realize that even assuming $N_{\mathbf{k}}=0$, the decay of a single axion on the entire universe would be enough to trigger the stimulated photon emission, due to the high occupation number of the axion condensate. For comparison, the stimulated decays of inflatons after inflations, discussed in preheating scenarios, needs vacuum fluctuations to provide the initial seed for the further decays $[47,48]$.

\section{Backreaction on axions}

We consider now the full solution of the kinetic equations of motion [Eq. (47)] taking into account also the evolution of the axion field. We consider the case of $\omega_{k}=m_{a} / 2$. In Fig. 3 shows the evolution of the axion occupation number for an initial $N_{a}=100$, as a function of the variable $s=\mu t$. We find that the axion condensate exhibits a periodic behavior, passing through minima of total depletion and maximum of repopulation (anticorrelated with the photon population) with a period $T=2 / \mu$. In order to develop an analytical interpretation of the results discussed before, we follow the treatment presented in [53] for a trilinear Hamiltonian, exhibiting spontaneous parametric down conversion. We consider the simplified version of the Hamiltonian, described by Eq. (48), adding also the kinetic term for photons and axions

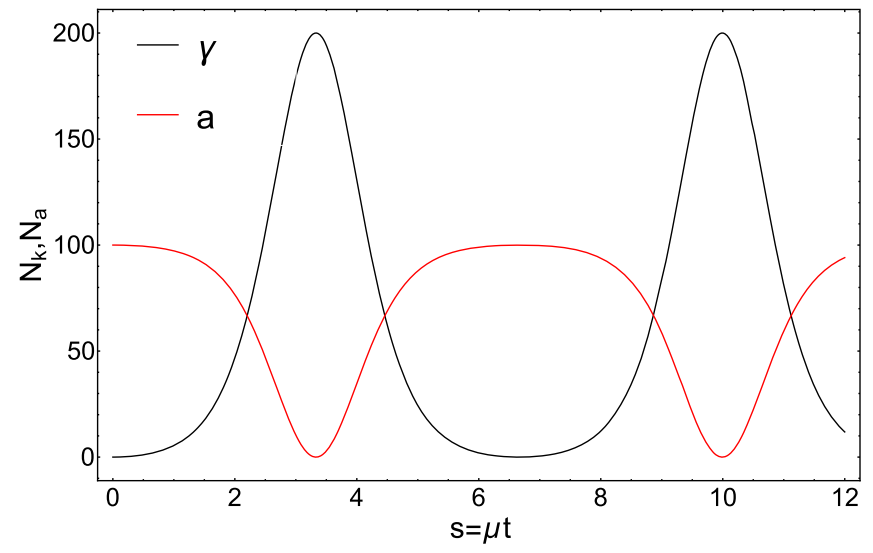

FIG. 3. Evolution of $N_{a}$ and $N_{\mathbf{k}}$ in function of $s=\mu t$ for the mode with $\omega_{k}=m_{a} / 2$ for an initial $N_{a}=100$. 


$$
\begin{aligned}
H_{\mathrm{int}} & =\kappa\left[b^{\dagger} c_{\mathbf{k}} c_{-\mathbf{k}}+b c_{\mathbf{k}}^{\dagger} c_{-\mathbf{k}}^{\dagger}\right], \\
H_{0} & =m_{a} b^{\dagger} b+\frac{m_{a}}{2}\left(c_{\mathbf{k}}^{\dagger} c_{\mathbf{k}}+c_{-\mathbf{k}}^{\dagger} c_{-\mathbf{k}}\right) .
\end{aligned}
$$

We introduce the operators

$$
\begin{aligned}
& R^{+}=b^{\dagger} c_{-\mathbf{k}}, \quad|R|^{2}=R(R+1), \\
& R_{3}=\frac{1}{2}\left(b^{\dagger} b-c_{-\mathbf{k}}^{\dagger} c_{-\mathbf{k}}\right), \quad R=\frac{1}{2}\left(b^{\dagger} b+c_{-\mathbf{k}}^{\dagger} c_{-\mathbf{k}}\right),
\end{aligned}
$$

which satisfy the algebra of the angular momentum operators. Using these latter the Hamiltonian reads

$$
\begin{aligned}
H_{\mathrm{int}} & =\kappa\left[R^{+} c_{\mathbf{k}}+R^{-} c_{\mathbf{k}}^{\dagger}\right], \\
H_{0} & =m_{a}\left(R_{3}+c_{\mathbf{k}}^{\dagger} c_{\mathbf{k}}\right),
\end{aligned}
$$

after subtracting a constant term

$$
C=m_{a}\left(\frac{1}{2} b^{\dagger} b+c_{-\mathbf{k}}^{\dagger} c_{-\mathbf{k}}-\frac{1}{2} c_{\mathbf{k}}^{\dagger} c_{\mathbf{k}}\right)
$$

from the free Hamiltonian. The equations of motion have the form

$$
\begin{aligned}
& \dot{c}_{\mathbf{k}}=-i \kappa R^{-}, \quad \dot{R}^{-}=2 i \kappa c_{\mathbf{k}} R_{3}, \\
& \dot{R}_{3}=i \kappa\left(c_{\mathbf{k}}^{\dagger} R^{-}-c_{\mathbf{k}} R^{+}\right) .
\end{aligned}
$$

We now make the mean field approximation which should be justified for reasonably large photon occupation numbers and we obtain

$$
\dot{\gamma}_{\mathbf{k}}=\kappa r_{1}, \quad \dot{r}_{1}=2 \kappa \gamma_{\mathbf{k}} r_{3}, \quad \dot{r}_{3}=-2 \kappa \gamma_{\mathbf{k}} r_{1},
$$

where $-i \gamma_{\mathbf{k}}=\left\langle c_{\mathbf{k}}\right\rangle, r_{1}=\left\langle R^{ \pm}\right\rangle$. The constants of motion are

$$
r_{3}^{2}+r_{1}^{2}=J^{2} ; \quad M=\gamma_{\mathbf{k}}^{2}+r_{3},
$$

and

$$
\dot{\gamma}_{\mathbf{k}}^{2}=\kappa^{2} r_{1}^{2}=J^{2}-\left(M-\gamma_{\mathbf{k}}^{2}\right)^{2} .
$$

Introducing the polar coordinates

$$
r_{1}=J \sin \theta, \quad r_{3}=J \cos \theta,
$$

one gets

$$
\dot{\theta}=2 \kappa \gamma_{\mathbf{k}} \rightarrow \ddot{\theta}=2 \kappa^{2} J \sin \theta .
$$

The parameters $\mathrm{J}$ and $\theta$ can be read as

$$
\begin{gathered}
J=\frac{1}{2}\left(N_{a}+N_{-\mathbf{k}}\right)=r, \\
\theta=\operatorname{arctg}\left(\frac{r_{1}}{r_{3}}\right)=\operatorname{arctg}\left(\frac{2 \sqrt{N_{a} N_{-\mathbf{k}}}}{N_{a}-N_{-\mathbf{k}}}\right) .
\end{gathered}
$$

Equation (61) represents the evolution of an inverted pendulum with frequency $\sqrt{2 J}$. The period of the pendulum is given by [55]

$$
T=\frac{4}{\sqrt{2 J}} K\left(\sin \left(\theta_{0} / 2\right)\right)
$$

where the function $K$ is the complete elliptical integral

$$
K(k)=\int_{0}^{\pi / 2} d \xi \frac{1}{\sqrt{1-k^{2} \sin ^{2} \xi}},
$$

and for an inverted pendulum $\theta_{0} \rightarrow \tilde{\theta}=\theta_{0}+\pi$, with $\theta_{0} \ll 1$ since initially $N_{-\mathbf{k}} \simeq 0$. Expanding the elliptical integral we obtain the period of the pendulum as

$$
T \simeq \frac{2}{\mu}\left|\ln \left(\frac{N_{-\mathbf{k}}}{N_{a}+N_{-\mathbf{k}}}\right)\right| \simeq \frac{2}{\mu} \ln N_{a}
$$

since $\cos ^{2}\left(\theta_{0} / 2\right)=\frac{1}{2}-\frac{1}{2}\left(N_{a}-N_{-\mathbf{k}}\right) /\left(N_{a}+N_{-\mathbf{k}}\right)$. Therefore we reproduce the result seen in Fig. 3 and we found a logarithmic correction associated with $N_{a}$ as predicted in [39]. It is interesting to notice that the behavior of inverted pendulum of the stimulated emission of photons has a nice analogy with the behavior of a dense neutrino gas under flavor conversions [56].

Next we consider the fact that axions may decay in different directions. It is easy to realize that increasing the number of directions one reduces the logarithmic delay in the period [39]

$$
T=\frac{2}{\mu}\left[\ln \left(\frac{N_{a}}{N_{d}}\right)\right] .
$$

Indeed, considering our system enclosed in a box of size $L$, so that the wave numbers are quantized as

$$
\mathbf{k}=\frac{2 \pi}{L} \mathbf{n}
$$

where $\mathbf{n}$ is a vector of integers. The number of possible directions, given the constraint $\omega_{k}=m_{a} / 2$, is 


$$
\begin{aligned}
N_{d} & =\int d^{3} \mathbf{n} \delta\left(n-\frac{L m_{a}}{4 \pi}\right) \\
& =4 \pi \int d n n^{2} \delta\left(n-\frac{L m_{a}}{4 \pi}\right) \\
& =\left.4 \pi n^{2}\right|_{\omega_{k}=m_{a} / 2}=\frac{1}{4 \pi} L^{2} m_{a}^{2},
\end{aligned}
$$

where $n=|\mathbf{n}|$. Finally we relax the assumption that $\omega_{k}=$ $m_{a} / 2$ and we consider possible unstable modes inside the band of Eq. (45). The number of modes in this band is

$$
\frac{2 \pi}{L} \Delta n=2 \mu \rightarrow \Delta n=\frac{\mu L}{\pi} .
$$

Then, the effective axion number in Eq. (66) is reduced by another factor $N_{t}=\mu L / \pi$. Therefore, including also this effect we find

$$
T=\frac{2}{\mu}\left[\ln \left(\frac{N_{a}}{N_{d} N_{t}}\right)\right] .
$$

\section{COSMOLOGICAL APPLICATIONS}

We analyze here the possibility of the axion-photon instability in two cosmological environments: the early Universe at recombination and a localized high-density axion clump.

\section{A. Early Universe}

At first we consider an homogeneous axion field at recombination (at redshift $z=1100$ ). The density of axion cold dark matter is given by [15]

$$
\begin{aligned}
n_{a}\left(t_{0}\right) & =\left(\frac{4 \times 10^{47}}{c m^{3}}\right)\left(\frac{F_{a}}{10^{12} \mathrm{GeV}}\right)^{5 / 3}\left(\frac{a\left(t_{1}\right)}{a\left(t_{0}\right)}\right)^{3} \\
& =\left(\frac{1.1 \times 10^{14}}{\mathrm{~cm}^{3}}\right)\left(\frac{g_{a \gamma}}{10^{-11} \mathrm{GeV}^{-1}}\right)^{-5 / 3},
\end{aligned}
$$

where $a\left(t_{1}\right)$ corresponds to the scale factor at the QCD phase transition $\left(T_{1}=1 \mathrm{GeV}\right)$, when axions are produced, and we expressed the axion-photon coupling as [14]

$$
g_{a \gamma}=\frac{1}{2 \pi} \frac{\alpha}{F_{a}}
$$

where $\alpha$ is the fine-structure constant. In the numerical evaluation we used a photon-axion coupling possible for generic ALP dark matter. For QCD axions, one should consider a coupling smaller by three order of magnitues. The typical exponential growth rate of photons from stimulated axion decays is $\mu \sim g_{a \gamma} \sqrt{m_{a} n_{a}}$. In order to have an efficient production, at first one should require that $\mu>H$, where $H$ is the Hubble parameter. In the matter-dominated era $H=$ $H_{0}(1+z)^{3 / 2}$ with $H_{0}=68 \mathrm{kms}^{-1} \mathrm{Mpc}^{-1}=2.2 \times 10^{-18} \mathrm{~s}^{-1}$
The photon plasma frequency can be neglected after recombination because $\omega_{p}<10^{-11} \mathrm{eV} \ll m_{a}$. One finds

$$
\begin{aligned}
& \mu / H \simeq\left(\frac{4 \times 10^{47}}{\mathrm{~cm}^{3}}\right)^{1 / 2}\left(\frac{F_{a}}{10^{12} \mathrm{GeV}}\right)^{-1 / 6}\left(\frac{a\left(t_{1}\right)}{a\left(t_{0}\right)}\right)^{3 / 2} \\
& \frac{\alpha}{2 \pi} 10^{-12} \mathrm{GeV}^{-1}\left(\frac{m_{a}}{\mu \mathrm{eV}}\right)^{1 / 2} \mu \mathrm{eV}^{1 / 2} \frac{1}{H_{0}(1+z)^{3 / 2}} \\
&=\left(\frac{F_{a}}{10^{12} \mathrm{GeV}}\right)^{-1 / 6}\left(\frac{m_{a}}{\mu \mathrm{eV}}\right)^{1 / 2} \frac{1.4 \times 10^{9}}{(1+z)^{3 / 2}} \\
&= 2.0 \times 10^{10}\left(\frac{g_{a \gamma}}{10^{-11} \mathrm{GeV}^{-1}}\right)^{1 / 6} \\
& \times\left(\frac{m_{a}}{10^{-5} \mathrm{eV}}\right)^{1 / 2}(1+z)^{-3 / 2}
\end{aligned}
$$

This first condition is satisfied. However, as noticed already in seminal papers $[57,58]$ the expansion of the Universe would redshift momenta of previously created photons taking them out the instability layer. Therefore, the occupation numbers relevant for the stimulated conversions are smaller than in a static case. The number of photons in the instability band is given by $\Delta k / m_{a} \sim \mu / m_{a}$. Therefore in order to have an efficient photon growth one should require $[57,58]$

$$
\frac{\mu}{m_{a}} \frac{\mu}{H} \gg 1
$$

However, in our case we numerically obtain

$$
\begin{aligned}
\frac{\mu}{H} \frac{\mu}{m_{a}} & =2.1 \times 10^{-9}\left(\frac{F_{a}}{10^{12} \mathrm{GeV}}\right)^{-1 / 3}(1+z)^{-3 / 2} \\
& =4.2 \times 10^{-8}\left(\frac{g_{a \gamma}}{10^{-11} \mathrm{GeV}^{-1}}\right)^{1 / 3}(1+z)^{-3 / 2},
\end{aligned}
$$

Therefore, based on this argument, already seminal papers $[57,58]$ excluded a sizable decay of the axion dark matter (see, however, the recent discussion in [40]). We notice that a similar analysis has been presented in the context of inflaton reheating during reheating [47-49]. A formal derivation of the Mathieu equation characterizing the axion instability [Eq. (14)] in the expanding Universe and the effect of the redshift is presented in Appendix A.

\section{B. Axion clumps}

If the Peccei-Quinn symmetry is broken after inflation, the axion field may present large inhomogeneities from one Hubble patch to another one. In this case gravitational interaction would organize axion dark matter in the form of localized clumps, known as Bose stars, oscillons, that can arrange themselves in miniclusters [59]. These dense axion objects have motivated investigations on the stimulated decay of axions. As an example, we consider the 
clump model presented in [60] which has the following parameters

$$
\begin{aligned}
N_{\max } & =1.6 \times 10^{56}\left(\frac{m_{a}}{10^{-5} \mathrm{eV}}\right)^{-2}\left(\frac{g_{a \gamma}}{10^{-11} \mathrm{GeV}^{-1}}\right)^{-1}, \\
R_{\min } & =6.5 \times 10^{5} \mathrm{~km}\left(\frac{m_{a}}{10^{-5} \mathrm{eV}}\right)^{-1}\left(\frac{g_{a \gamma}}{10^{-11} \mathrm{GeV}^{-1}}\right), \\
\rho_{\max } & =2.4 \times 10^{-18} \mathrm{kgcm}^{-3}\left(\frac{m_{a}}{10^{-5} \mathrm{eV}}\right)^{2}\left(\frac{g_{a \gamma}}{10^{-11} \mathrm{GeV}^{-1}}\right)^{-4},
\end{aligned}
$$

where $N_{\max }$ is the maximum axion number, $R_{\min }$ is the minimum radius, and $\rho_{\max }$ is the maximum axion density to guarantee stable configurations. For these parameter one obtains as photon exponential growth rate

$$
\begin{aligned}
\mu & =\frac{1}{\sqrt{8}} g_{a \gamma} \rho_{\max }^{1 / 2} \\
& =1.7 \times 10^{-9} \mathrm{~km}^{-1}\left(\frac{m_{a}}{10^{-5} \mathrm{eV}}\right)\left(\frac{g_{a \gamma}}{10^{-11} \mathrm{GeV}^{-1}}\right)^{-1} .
\end{aligned}
$$

Note that for an axion clump the effect of the redshift due to Universe expansion is negligible in comparison to the gravitational field of the clump itself. This growth rate $\mu$ should be compared with a typical size $R$ of the clump [30]. For an homogeneous axion clump one should require $\mu R \gg 1$ in order to achieve a significant stimulated photon emission with an axion feedback. In the inhomogeneous case, as long as the growth rate is adiabatic

$$
\left|\frac{1}{\mu} \frac{d \mu}{d r}\right| \ll \mu,
$$

one would get as e-folding factor for the photon amplitude $\int \mu(r) d r$ (see Eqs. (B31)-(B32) in [48]). However, from the adiabaticity condition since $d \mu / d r \sim \mu / R$ one gets once more $\mu R \gg 1$ for a sizable effect, such that our rough estimate is justified. Therefore, for our benchmark axion parameters, in order to get $\mu R \gg 1$ one should have an axion minicluster of size $R \gtrsim 10^{3} R_{\min }$. In this situation the minicluster might even "explode" under photon emission [35]. We also mention that in [31] it has been estimated that axion minicluster might reach an extension $R$ for which $\mu R \gtrsim 1$ for $g_{a \gamma} \gtrsim 2 \times 10^{-13}\left[m_{a} /\left(10^{-5} \mathrm{eV}\right)\right]^{1 / 3} \mathrm{GeV}^{-1}$.

\section{Constraints}

We note that in general globally only a very small fraction $\Delta N_{a} / N_{a}$ of axion dark matter can be converted into photons because the energy in radiation today is much small than the rest mass density in dark matter, $\Omega_{\mathrm{dm}}$ in units of the critical density [26]. More quantitatively, if axions form a fraction $f_{\mathrm{dm}}$ of the cold dark matter, then

$$
\frac{\Delta N_{a}}{N_{a}} f_{\mathrm{dm}} \lesssim \frac{\Omega_{\gamma}\left(m_{a} / 2\right)}{\Omega_{\mathrm{dm}}} \frac{\Delta \nu}{\nu},
$$

where $\Omega_{\gamma}\left(m_{a} / 2\right)$ is the photon energy density per logarithmic photon energy in terms of the critical density and $\Delta \nu / \nu$ is the maximum of the predicted axion conversion line width and experimental relative frequency resolution, provided that no line is detected. For frequencies $\nu \lesssim 1 \mathrm{GHz}$ the energy density in the universal radio background is of the order of $2 \times 10^{-8} \mathrm{eV} \mathrm{cm}^{-3}$, see e.g., Ref. [61], which should be compared with the dark matter density $\Omega_{\mathrm{dm}} \rho_{\text {crit }} \simeq 10^{3} \mathrm{eV} \mathrm{cm}^{-3}$. Therefore, for $m_{a} \sim \mu \mathrm{eV}, \Omega_{\gamma}\left(m_{a} / 2\right) / \Omega_{\mathrm{dm}} \sim 10^{-10}$.

The number of converted axions will be $\Delta N_{a} \sim$ $N_{d} N_{t}\left[N_{m_{a} / 2}(T)-N_{m_{a} / 2}(0)\right]$, where $N_{k}(t)$ is given by Eq. (51), $N_{d} N_{t}$ is the number of photon modes into which the axion can decay, see Eq. (69)-(70), and $T$ is the timescale over which the resonance acts which is typically of the order the size $R$ of the axion system. Since $N_{a}$ is a huge number, a significant constraint is only obtained for $\mu T \gg 1$. The above constraint thus simplifies to

$$
\mu T \lesssim \frac{1}{2} \ln \left[\frac{\Omega_{\gamma}\left(m_{a} / 2\right)}{f_{\mathrm{dm}} \Omega_{\mathrm{dm}}} \frac{N_{a}}{N_{d} N_{t}\left(N_{m_{a} / 2}(0)+1\right)} \frac{\Delta \nu}{\nu}\right] .
$$

This is generally fulfilled for the cosmological case and the axion clumps discussed above. It is, however, clear that Eq. (80) excludes that a significant fraction of axions is in the form of structures satisfying $\mu T \gg 1$, independent of any external photon field. This could constrain axion miniclusters [31].

Equation (80) is different from constraints discussed [26] which considered the relative enhancement of a diffuse photon background impinging on an axion condensate in the Mathieu regime.

\section{CONCLUSIONS}

In this work we revisited the stimulated decay of dark matter axion condensates into photon pairs. In particular, we have considered the decay into a narrow instability width around photon energies $\omega \approx m_{a} / 2$ where one can have an exponential growth of the photon occupation number. This effect has been often interpreted as a parametric resonance of photons from the axion-photon coupling, treating the photon field as a classical one and limiting the solution to the initial exponential growth. We have analyzed in details this effect presenting a mean field solution of the axion-photon kinetic equations, in terms of number of photons and pair correlations. We have studied the limit of negligible axion depletion, recovering the known instability. Moreover, we extended the results including a possible depletion of the axion field. Interestingly, in the mean field approximation we find that 
the solution can be interpreted in terms of the periodic motion of an inverted pendulum.

The instability of axion condensates to decay into photons is relevant for cosmological environments. In particular, we considered the case of a homogeneous axion field at recombination and a localized axion clump. In the first case, we confirm that due to the expansion of the Universe the photon production would not be efficient, since modes will quickly be redshifted away from the instability band. Conversely, in a localized clump one might have relevant photon production. We also discussed constraints that could result from overproduction of the diffuse photon background by axion-photon conversion which exclude that any significant fraction of dark matter axions is in structures with $\mu T \gg 1$. This can concern the case of axion miniclusters of relatively large size for which the feedback can be significant. Further studies are necessary to investigate this case. Other systems to which our formalism can be applied include the case of evaporation of axion stars that can lead to fast radio bursts [35], and stimulated axion decay in superradiant clouds around primordial black holes [36-38]. The study of these interesting systems is left to a future work.

\section{ACKNOWLEDGMENTS}

A. M. thanks Raymond Sawyer and Javier Redondo for stimulating discussions. We thank Georg Raffelt for useful comments on the manuscript. We acknowledge Cannon Vogel for discussions of the Kapitza pendulum. The work of P.C. and A. M. is partially supported by the Italian Istituto Nazionale di Fisica Nucleare (INFN) through the "Theoretical Astroparticle Physics" project and by the research Grant No. 2017W4HA7S "NAT-NET: Neutrino and Astroparticle Theory Network" under the program PRIN 2017 funded by the Italian Ministero dell'Università e della Ricerca (MUR). The work of G. S. was supported under Germany's Excellence Strategy-EXC 2121 "Quantum Universe"-39083306. We thank Cannon Vogel for discussions of the Kapitza pendulum.

Note added.-After our preprint appeared, in [45] a similar study has been presented. The two works reach similar conclusions.

\section{APPENDIX: AXION INSTABILITY IN THE EXPANDING UNIVERSE}

In this Appendix we generalize the treatment to the axion-photon instability in the case of the expanding Universe, characterized by the Friedman-RobertsonWalker (FRW) metric, whose line element reads

$$
d s^{2}=d t^{2}-a(t)^{2} g_{i j} d x^{i} d x^{j},
$$

where $a(t)$ is the scale factor of the Universe, $g_{i j}$ is the three metric for a flat three-space.

The ALP-photon Lagrangian is

$$
\mathcal{L}=-\frac{1}{4} g^{\mu \alpha} g^{\nu \beta} F_{\mu \nu} F_{\alpha \beta}-\frac{g_{a \gamma}}{4} \phi \frac{1}{2} \frac{\epsilon^{\mu \nu \alpha \beta}}{\sqrt{-g}} F_{\mu \nu} F_{\alpha \beta},
$$

and the equations of motion for the electromagnetic field are given by

$$
\partial_{\mu} \frac{\partial(\sqrt{-g} \mathcal{L})}{\partial\left(\partial_{\mu} A_{\nu}\right)}=\frac{\partial(\sqrt{-g} \mathcal{L})}{\partial A_{\nu}}
$$

Working in the Coulomb gauge $\left(\partial_{i} A^{i}=0\right)$ and assuming an homogeneous axion field, one gets the modified Maxwell equations

$$
\left(\frac{\partial^{2}}{\partial t^{2}}+H \frac{\partial}{\partial t}-\frac{\nabla^{2}}{a^{2}}\right) \mathbf{A}-g_{a \gamma} \dot{\phi}(t) \frac{\nabla}{a} \times \mathbf{A}=0
$$

where we introduced the Hubble parameter $H=\dot{a} / a{ }^{1}$

Expanding the electromagnetic field in plane wave as in Eq. (13) and considering that the axion field of Eq. (11) scales with Universe expansion as $a^{-3 / 2}$ we get the following equation of motion for the two polarization states of photons (see also [45]):

$$
\frac{d^{2} c_{\mathbf{k}, \pm}}{d x^{2}}+\frac{2 H}{m_{a}} \frac{d c_{\mathbf{k}, \pm}}{d x}+\left(A_{\mathbf{k}} \mp 2 q \cos x\right) c_{\mathbf{k}, \pm}=0,
$$

where $x=m_{a} t / 2-\pi / 2, \quad A_{\mathbf{k}}=4|\mathbf{k}|^{2} / m_{a}^{2} a^{2} \quad$ and $q=$ $2 g_{a \gamma} \phi_{0}|\mathbf{k}| / m_{a} a^{5 / 2}$. The previous equation is consistent with Eq. (A1) of Ref. [45]. In the case $H \ll m_{a}$, which is satisfied in the situations we consider, the friction term can be neglected and one obtains a Mathieu equation, as in our Eq. (14). A discussion of the solution of this equation is given in $[49,62]$ to which we address the interested readers for further details. An important feature of the solution of this equation is the presence of an exponential instability $c_{\mathbf{k}, \pm} \sim \exp (\tilde{\mu} x)$. In the case of a narrow resonance $q \ll 1$, the instability occurs in narrow bands around $A_{\mathbf{k}}=n^{2}$, with $n=1,2, \ldots$ Each band in momentum space has width $\Delta k \sim q^{n}$, so for $q<1$ the widest and most important instability band is the first one, $A_{\mathbf{k}} \sim 1 \pm q$, where the maximum growth rate is $\tilde{\mu}=q / 2$. In our specific case we find as resonance frequency $k / a=m_{a} / 2$, and growth rate

$$
\mu=\tilde{\mu} \frac{m_{a}}{2}=\frac{g_{a \gamma} \phi_{0} k}{2 a^{5 / 2}}
$$

\footnotetext{
${ }^{1}$ Note that in terms of conformal time $d \eta=d t / a$ and comoving coordinates the wave operator in Eq. (A4) can be written as $\left(\frac{\partial^{2}}{\partial \eta^{2}}-\nabla^{2}\right)$.
} 
The edges of the instability band are given by

$$
\frac{\Delta k}{a}=2 \mu \text {. }
$$

As discussed in [49] in the context of cosmological preheating an important mechanism which can prevent the axion instability from being efficient is the redshift of momenta $k$ away from the instability band. The total width of the instability band is $\sim \frac{1}{2} q m_{a}$. The time $\Delta t$ during which a given mode remains within this band can be estimated as $q H^{-1}$. During this time the number of photons in growing modes increases as $\exp \left(\frac{q^{2} m}{2 H}\right)$. This leads to an efficient growth of photons only if

$$
q^{2} m \gtrsim H
$$

that corresponds to the condition we gave in Eq. (74).
[1] R. D. Peccei and H. R. Quinn, $C P$ Conservation in the Presence of Instantons, Phys. Rev. Lett. 38, 1440 (1977).

[2] R. D. Peccei and H. R. Quinn, Constraints imposed by $C P$ conservation in the presence of instantons, Phys. Rev. D 16, 1791 (1977).

[3] S. Weinberg, A New Light Boson?, Phys. Rev. Lett. 40, 223 (1978).

[4] F. Wilczek, Problem of Strong $P$ and $T$ Invariance in the Presence of Instantons, Phys. Rev. Lett. 40, 279 (1978).

[5] G. G. di Cortona, E. Hardy, J. Pardo Vega, and G. Villadoro, The QCD axion, precisely, J. High Energy Phys. 01 (2016) 034.

[6] S. Borsanyi et al., Calculation of the axion mass based on high-temperature lattice quantum chromodynamics, Nature (London) 539, 69 (2016).

[7] P. Sikivie, Experimental Tests of the Invisible Axion, Phys. Rev. Lett. 51, 1415 (1983); Erratum, Phys. Rev. Lett. 52, 695 (1984).

[8] G. Raffelt and L. Stodolsky, Mixing of the photon with low mass particles, Phys. Rev. D 37, 1237 (1988).

[9] A. A. Anselm, Experimental test for arion photon oscillations in a homogeneous constant magnetic field, Phys. Rev. D 37, 2001 (1988).

[10] G. G. Raffelt, Astrophysical axion bounds, Lect. Notes Phys. 741, 51 (2008).

[11] I. G. Irastorza and J. Redondo, New experimental approaches in the search for axion-like particles, Prog. Part. Nucl. Phys. 102, 89 (2018).

[12] P. Sikivie, Axion cosmology, Lect. Notes Phys. 741, 19 (2008).

[13] A. Ringwald, Alternative dark matter candidates: Axions, Proc. Sci., NOW2016 (2016) 081 [arXiv:1612.08933].

[14] P. Arias, D. Cadamuro, M. Goodsell, J. Jaeckel, J. Redondo, and A. Ringwald, WISPy cold dark matter, J. Cosmol. Astropart. Phys. 06 (2012) 013.

[15] P. Sikivie and Q. Yang, Bose-Einstein Condensation of Dark Matter Axions, Phys. Rev. Lett. 103, 111301 (2009).

[16] S. Davidson and M. Elmer, Bose Einstein condensation of the classical axion field in cosmology?, J. Cosmol. Astropart. Phys. 12 (2013) 034.

[17] S. Davidson, Axions: Bose einstein condensate or classical field?, Astropart. Phys. 65, 101 (2015).
[18] N. Du et al. (ADMX Collaboration), A Search for Invisible Axion Dark Matter with the Axion Dark Matter Experiment, Phys. Rev. Lett. 120, 151301 (2018).

[19] A. Caldwell, G. Dvali, B. Majorovits, A. Millar, G. Raffelt, J. Redondo, O. Reimann, F. Simon, and F. Steffen (MADMAX Working Group), Dielectric Haloscopes: A New Way to Detect Axion Dark Matter, Phys. Rev. Lett. 118, 091801 (2017).

[20] M. Lawson, A. J. Millar, M. Pancaldi, E. Vitagliano, and F. Wilczek, Tunable Axion Plasma Haloscopes, Phys. Rev. Lett. 123, 141802 (2019).

[21] J. E. Kim, Weak Interaction Singlet and Strong $C P$ Invariance, Phys. Rev. Lett. 43, 103 (1979).

[22] M. A. Shifman, A. I. Vainshtein, and V. I. Zakharov, Can confinement ensure natural $C P$ invariance of strong interactions?, Nucl. Phys. B166, 493 (1980).

[23] G. G. Raffelt, Axions: Motivation, limits and searches, J. Phys. A 40, 6607 (2007).

[24] A. Caputo, M. Regis, M. Taoso, and S. J. Witte, Detecting the stimulated decay of axions at radiofrequencies, J. Cosmol. Astropart. Phys. 03 (2019) 027.

[25] A. Caputo, C. P. Garay, and S. J. Witte, Looking for axion dark matter in dwarf spheroidals, Phys. Rev. D 98, 083024 (2018); Erratum, Phys. Rev. D 99, 089901 (2019).

[26] G. Sigl and P. Trivedi, Axion condensate dark matter constraints from resonant enhancement of background radiation, arXiv:1907.04849.

[27] A. Arza and P. Sikivie, Production and Detection of An Axion Dark Matter Echo, Phys. Rev. Lett. 123, 131804 (2019).

[28] R. A. Battye, B. Garbrecht, J. I. McDonald, F. Pace, and S. Srinivasan, Dark matter axion detection in the radio/mmwaveband, arXiv:1910.11907.

[29] D. Espriu and A. Renau, Photon propagation in a cold axion background with and without magnetic field, Phys. Rev. D 85, 025010 (2012).

[30] M. P. Hertzberg and E. D. Schiappacasse, Dark matter axion clump resonance of photons, J. Cosmol. Astropart. Phys. 11 (2018) 004.

[31] A. Arza, Photon enhancement in a homogeneous axion dark matter background, Eur. Phys. J. C 79, 250 (2019).

[32] E. Braaten, A. Mohapatra, and H. Zhang, Emission of photons and relativistic axions from axion stars, Phys. Rev. D 96, 031901 (2017). 
[33] I. I. Tkachev, An axionic laser in the center of a galaxy?, Phys. Lett. B 191, 41 (1987).

[34] T. W. Kephart and T. J. Weiler, Stimulated radiation from axion cluster evolution, Phys. Rev. D 52, 3226 (1995).

[35] I. I. Tkachev, Fast radio bursts and axion miniclusters, Pis'ma Zh. Eksp. Teor. Fiz. 101, 3 (2015) [JETP Lett. 101, 1 (2015)].

[36] J. G. Rosa and T. W. Kephart, Stimulated Axion Decay in Superradiant Clouds around Primordial Black Holes, Phys. Rev. Lett. 120, 231102 (2018).

[37] T. Ikeda, R. Brito, and V. Cardoso, Blasts of Light from Axions, Phys. Rev. Lett. 122, 081101 (2019).

[38] M. Boskovic, R. Brito, V. Cardoso, T. Ikeda, and H. Witek, Axionic instabilities and new black hole solutions, Phys. Rev. D 99, 035006 (2019).

[39] R. F. Sawyer, Axion, photon-pair mixing in models of axion dark matter, arXiv:1809.01183.

[40] R. F. Sawyer, Quantum break in models of axion dark matter, arXiv:1908.04298.

[41] V. Mukhanov, Physical Foundations of Cosmology (Cambridge University Press, Cambridge, England, 2005).

[42] F. Wilczek, Two Applications of Axion Electrodynamics, Phys. Rev. Lett. 58, 1799 (1987).

[43] E. Masaki, A. Aoki, and J. Soda, Stability of axion dark matter-photon conversion, Phys. Rev. D 101, 043505 (2020).

[44] M. A. Fedderke, P. W. Graham, and S. Rajendran, Axion dark matter detection with CMB polarization, Phys. Rev. D 100, 015040 (2019).

[45] G. Alonso-Álvarez, R. S. Gupta, J. Jaeckel, and M. Spannowsky, On the wondrous stability of ALP dark matter, arXiv:1911.07885.

[46] F. M. Phelps III and J. H. Hunter, Jr., An analytical solution of the inverted pendulum, Am. J. Phys. 33, 285 (1965).

[47] J. H. Traschen and R. H. Brandenberger, Particle production during out-of-equilibrium phase transitions, Phys. Rev. D 42, 2491 (1990).
[48] Y. Shtanov, J.H. Traschen, and R. H. Brandenberger, Universe reheating after inflation, Phys. Rev. D 51, 5438 (1995).

[49] L. Kofman, A. D. Linde, and A. A. Starobinsky, Towards the theory of reheating after inflation, Phys. Rev. D 56, 3258 (1997).

[50] P. Adshead, J. T. Giblin, T. R. Scully, and E. I. Sfakianakis, Gauge-preheating and the end of axion inflation, J. Cosmol. Astropart. Phys. 12 (2015) 034.

[51] P. Adshead, J. T. Giblin, and Z. J. Weiner, Gravitational waves from gauge preheating, Phys. Rev. D 98, 043525 (2018).

[52] B. R. Mollow and R. J. Glauber, Quantum theory of parametric amplification. I, Phys. Rev. 160, 1076 (1967).

[53] R. Bonifacio and G. Preparata, Coherent spontaneous emission, Phys. Rev. A 2, 336 (1970).

[54] A. Bandilla, G. Drobny, and I. Jex, Parametric downconversion and maximum pump depletion, J. Opt. B 2, 265 (2000).

[55] L. D. Landau and E. M. Lifshitz, Mechanics, 3rd ed. (Pergamon, Oxford, 1976).

[56] S. Hannestad, G. G. Raffelt, G. Sigl, and Y. Y. Y. Wong, Self-induced conversion in dense neutrino gases: Pendulum in flavour space, Phys. Rev. D 74, 105010 (2006); Erratum, Phys. Rev. D 76, 029901 (2007).

[57] J. Preskill, M. B. Wise, and F. Wilczek, Cosmology of the invisible axion, Phys. Lett. B 120, 127 (1983).

[58] L. F. Abbott and P. Sikivie, A cosmological bound on the invisible axion, Phys. Lett. B 120, 133 (1983).

[59] E. W. Kolb and I. I. Tkachev, Axion Miniclusters and Bose Stars, Phys. Rev. Lett. 71, 3051 (1993).

[60] E. D. Schiappacasse and M. P. Hertzberg, Analysis of dark matter axion clumps with spherical symmetry, J. Cosmol. Astropart. Phys. 01 (2018) 037; 03 (2018) E01.

[61] R. Hill, K. W. Masui, and D. Scott, The spectrum of the universe, Appl. Spectrosc. 72, 663 (2018).

[62] N. W. Mac Lachlan, Theory and Application of Mathieu functions (Dover, New York, 1961). 